\title{
PALEO
}

Revue d'archéologie préhistorique

$22 \mid 2011$

Varia

\section{Spécificités et parenté du dispositif pariétal de l'abri Reverdit (Sergeac, Dordogne)}

L'apport de l'étude des blocs ornés de la collection Delage

Specificity and analogy in Reverdit rock art: the contribution of the decorated blocks from the Delage collection

\section{Camille Bourdier}

\section{OpenEdition}

\section{Journals}

Édition électronique

URL : http://journals.openedition.org/paleo/2070

DOI : $10.4000 /$ paleo.2070

ISSN : 2101-0420

Éditeur

SAMRA

Édition imprimée

Date de publication : 1 décembre 2011

Pagination : 53-68

ISSN : 1145-3370

\section{Référence électronique}

Camille Bourdier, «Spécificités et parenté du dispositif pariétal de l'abri Reverdit (Sergeac, Dordogne) », PALEO [En ligne], 22 | 2011, mis en ligne le 13 avril 2012, consulté le 07 juillet 2020. URL : http:// journals.openedition.org/paleo/2070 ; DOI : https://doi.org/10.4000/paleo.2070

\section{(c) (i) (3) $\Theta$}

PALEO est mis à disposition selon les termes de la licence Creative Commons Attribution - Pas d'Utilisation Commerciale - Pas de Modification 4.0 International. 


\title{
SPÉCIFICITÉS ET PARENTÉ DU DISPOSITIF PARIÉTAL DE L'ABRI REVERDIT (Sergeac, Dordogne) : l'apport de l'étude des blocs ornés de la collection Delage
}

\author{
Camille BOURDIER(1)
}

en hommage à Norbert AUJOULAT

\begin{abstract}
Résumé : Les blocs ornés viennent compléter les études menées sur les manifestations graphiques pariétales de l'abri Reverdit par des thématiques, des techniques et des conventions formelles distinctes de celles qui composent la frise in situ. Leur analyse met notamment en avant la coexistence de deux morphotypes de Bison et révèle de nouveaux parallèles avec les sculptures du Roc-aux-Sorciers, abri occupé et orné au Magdalénien moyen (15 000-14 000 BP). Les motifs cupulés soulignent, en outre, la singularité de ce dispositif pariétal.
\end{abstract}

Mots-clés : Reverdit, art pariétal, bloc, cupule, bison, Magdalénien.

Key-words: Reverdit, rock art, block, cupule, bison, Magdalenian.

\section{Abridged english version}

\section{Specificity and analogy in Reverdit rock art: the contribution of the decorated blocks from the Delage collection.}

Reverdit is one of the numerous rock-shelters dug in the cliffs of Castel-Merle vale in the Vézère valley, intensively occupied from the Mousterian period until the end of the Upper Palaeolithic (fig. 1). Facing east, it opens to the left bank, facing Castanet rock-shelter.

Early discovered (Reverdit 1878), the site is mainly excavated between 1911 and 1914 by F. Delage who identifies two archaeological layers, both dated from the Magdalenian III (Delage 1927, 1935). Between 1985 and 1987, D. Robin and A. Roussot investigate a residual fill at the south extremity of the site (Robin 1985, 1986a, 1986b, 1987). They identify three archaeological layers, equating their two lower layers to the layers discovered by F. Delage. In their opinion, the middle layer dates from the Middle Magdalenian, whereas the chrono-cultural attribution of the lower layer is more ambiguous (Middle Magdalenian? Lower Magdalenian?).

Since 2007, as part of our research on the rock-shelters occupied and sculpted, during the Middle Magdalenian, we have started the study of the rock art pieces which had not yet been analysed (Bourdier 2008). After the recognition of several successive graphic sets on the in situ frieze, a collaboration has begun on the revision of the archaeological material (lithic and bone industries, art mobilier, personal adornment), with still pending radiocarbon dating (Bourdier et al. soumis). Concurrently, we have followed up the rock art study with the observation of the decorated blocks from the Delage collection.

\section{1 - The other side of Reverdit rock art}

During his excavations, before the discovery of the in situ frieze, F. Delage finds 19 decorated blocks, which he briefly describes and from which he only gives three photographs (Delage 1935). Most of them come from the first excavations rubbles and are not situated in the stratigraphy. Seven have been identified with no doubt: six in the Musée national de Préhistoire, and 1 still stored in the shelter. All except one seem to be pieces fallen from the wall rather than a ground decoration (art semi-mobilier), as figures are incomplete and not centred on the rock slab (Tymula 2002). 


\section{2 - The abstract imagery}

Four blocks present a complex geometric decoration associating cupules and engravings (fig. 2 to 5 ). The patterns differ. The disposition of the cupules varies : aligned in one or two ranks, joined or broadly juxtaposed, they are sometimes linked with incisions. Their morphology also changes, in diameter as in depth. Thus, block \#1 combines a huge cupule with a regular flat bottom and four smaller cupules with rounded bottoms. Except for this big cupule, the natural or anthropic origin of these elements is not easy to determine because of their erosion. However, the relative homogeneity of their shape as well as the regularity of the spacing on each block would support an anthropic action, also considering that the rock is not very naturally cupuled. Moreover, picking marks can be seen very distinctly at the bottom of some cupules. The engravings also vary in width and depth. Maybe the Palaeolithics did not make all the cupules but used natural ones as patterns in association with engravings.

Thus, Reverdit rock art gathers figurative and abstract themes. Though surprising, the lack of abstract graphism on the shelter wall does not seem meaningful to us because of its strong alteration (Bourdier 2008). The hypothesis of a strict separation between an abstract expression on blocks and a figurative composition on the wall does not hold with the present data. The frequent interruption of the marks at the edges of the blocks, and the coexistence of figurative works on blocks (see below) support the hypothesis of pieces fallen from the wall.

Other blocks stored in the shelter show similar patterns, but they cannot be connected to these blocks described above (fig. 6). A broader vision of this type of graphism would be all the more necessary so as cupule patterns are known in Aurignacian and Gravettian sites from the same vale. Nevertheless, their structure seem different from the " bear spoors ", cultural mark of the Aurignacian and Gravettian sites around Sergeac and Les Eyzies (Delluc et Delluc 1991) (fig. 7). The hypothesis of decorated elements gathered in the nearby sites by the Magdalenians is not privileged, although it must not be completely rejected. At the end, the permanence of such patterns would not be that surprising as cupules are a universal feature in rock art.

\section{3 -The animal sculptures}

The identification of the animal themes is complicated by the subjects fragmentation. Stuck in the fill, the blocks have less suffered than the frieze from frost weathering, intensified in the shelter by the presence of a spring (Bourdier 2008). Their surface does not present the same physico-chemical moonmilk-type degradation. A bison lower part, a herbivore hindlegs and an equidae (?) head can be recognized (fig. 8 to 10). These themes are identical to the frieze, gathering a herbivore (equidae), a horse and three bisons (Bourdier op. cit.). Moreover, their sizes match (head length, flank thickness, body length). These two elements underline the homogeneity of the graphic set, in the themes represented as well as in the composition (common representation scale).

\section{4 - A complex graphic structuration: the bison figuration}

Even though the subjects fragmentation on the wall and on the blocks reduces our appreciation, the bison on block differs from the two individuals in situ, in its technical and formal aspects (fig. 12). The light low-relief $(2-3 \mathrm{~cm}) \mathrm{created}$ by a plane digging contrasts with the thick low-relief $(10 \mathrm{~cm})$ on the frieze, made by a deep and curved digging (Bourdier 2008). The two in situ representations are characterized by a strong hypertrophy of the forelegs, due to a high and wide square dorsal hump. Unfortunately, this discriminating element cannot be checked on the block figure. In return, chest and beard are clearly different: a curved chest coming down on a vertical triangular beard on the one hand (frieze), and on the other hand a rectilinear chest with the fanon stylised as a strip in relief, going up to an oval beard, pointed forward (block). Moreover, although the same volumes are highlighted (shoulder, arm), their treatments deeply diverge, with a strong curve on one side (frieze), and a very flat relief on the other side (block). Thus, two Bison morphotypes seem to coexist inside the graphic set. Unlike F. Delage's opinion, the lower part on the block would not correspond to the second bison of the frieze, reduced to its dorsal outline, even though their sizes match (Delage 1935). Besides, it is not obvious that its lower part had ever been sculptured as its dorsal outline is unfinished (Bourdier op. cit.).

The analysis on the in situ frieze revealed two successive graphic sets, illustrated by sculpture renewals (Bourdier op. cit.). From the first set only two fragmentary sculptures of bison (?) subsist. A second graphic set may have taken its place, associating two bison, a horse and a herbivore (horse?). This evolution would not result from a theme change as the bison remains, but maybe from a diversification with the introduction of the horse. Then, it could be explained by a transformation of the graphic conventions, technical and/or formal. As its technique and morphotype are different from the subjects of the frieze, the bison on block is particularly revealing. Does it show another period of decoration? This hypothesis has already been formulated by J.-J. Cleyet-Merle, S. Madeleine and Ph. Jugie who saw a more essential contrast between rock art (in situ frieze) and art semi-mobilier on blocks (Cleyet-Merle et al. 1994).

This bison exhibits numerous analogies with sculptures from Roc-aux-Sorciers (Angles-sur-l'Anglin, Vienne), a rock shelter occupied and decorated during the Middle Magdalenian between 15000 and 14000 BP (18 000 - 16500 cal. BP) (lakovleva, Pinçon 1997). Its comparison with one of the Roc-aux-Sorciers bison is very striking (fig. 12). Its outline is very similar: an oval beard pointed forwards, a coming-down stomach line with the sex, finely shaped legs with the knuckle bend indicated, pairs of legs in the same posture. Moreover, the stylised representation of the fanon as a strip in relief is one of the typical Rocaux-Sorciers bison. Finally, dimensions and proportions are very close. Reverdit bison fragmentation is all the more regrettable as the head and dorsal hump cannot be considered. 


\section{Conclusions}

The decorated blocks bring new elements of knowledge on Reverdit rock art, on its composition on the one hand with a new abstract theme register, on its structuration on the other hand with the coexistence of two bison morphotypes. The use of sculpture, the similarity of the figurative themes on the frieze and on the blocks as well as a common representation scale underline the cohesion of this art. The analogy with Roc-aux-Sorciers sculptures would give a chrono-cultural indication (Middle Magdalenian), without extending this attribution to the whole graphic set as it only regards a single figuration.

Reverdit est l'un des nombreux abris-sous-roche creusés dans les falaises du vallon de Castel-Merle en vallée Vézère, intensément occupés depuis le Moustérien jusqu'à la fin du Paléolithique supérieur (fig. 1). Orienté à l'est, l'abri développe sur la rive gauche, face à l'abri Castanet.

Mis au jour en 1878 par A. Reverdit auquel succédèrent plusieurs chercheurs dont M. Hardy et M. Castanet, le gisement est principalement connu par les travaux de F. Delage qui, entre 1911 et 1914 , fouille le remplissage résiduel de l'abri et le talus localisé à l'avant (Reverdit 1878, 1882 ; Hardy 1880 ; Delage 1927, 1935). II identifie deux couches archéologiques séparées par un éboulis, qu'il attribue toutes deux au Magdalénien III, en référence d'une part à l'industrie osseuse (" sagaies à base conique et à rainure simple ", " aiguilles fines », " bâtons de commandement », absence de harpons) et, d'autre part, à

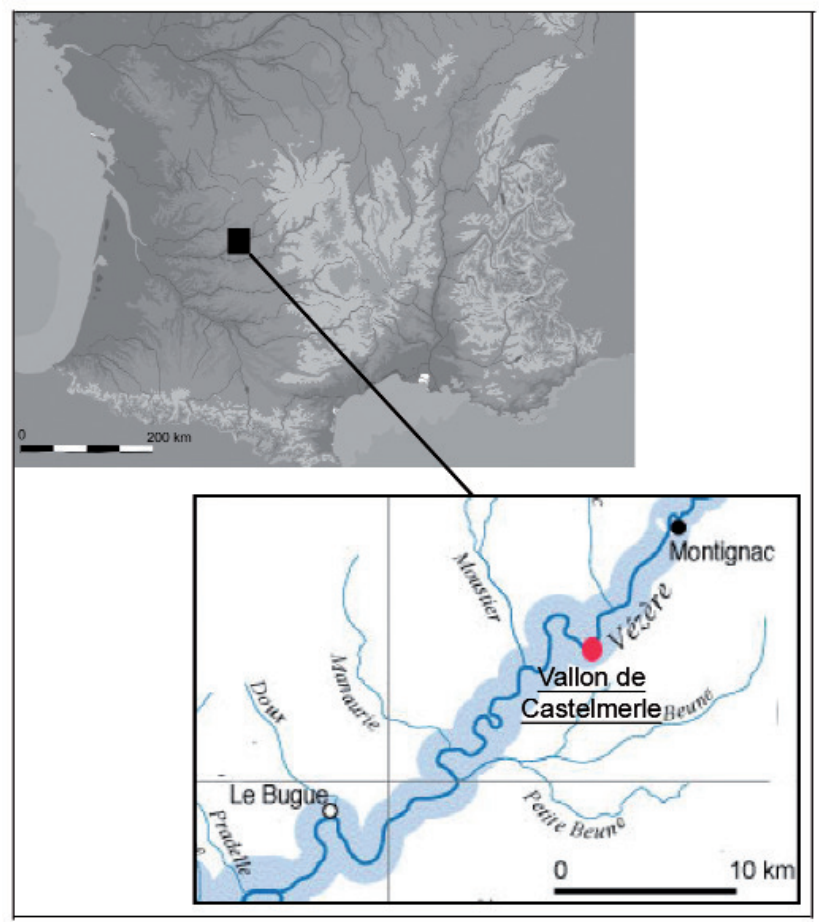

Figure 1 - Localisation de l'abri Reverdit.

Figure 1 - Reverdit rock-shelter situation. la frise sculptée découverte en 1923 par M. Castanet dans le fond de l'abri (Delage 1935). Lors de l'aménagement à vocation touristique du site, une reprise de la coupe stratigraphique sud-est réalisée par $D$. Robin et $A$. Roussot entre 1985 et 1987 (Robin 1985, 1986a, 1986b, 1987). Ils distinguent trois couches archéologiques (c.3, 5 et 6 ), associant les deux niveaux de base aux deux couches reconnues par F. Delage. Ils rapportent au Magdalénien moyen la couche 5 (fragments de baguette demi-rondes), mais l'attribution chrono-culturelle de la couche 6 est moins assurée. Cette coupe a récemment fait l'objet d'un examen taphonomique (Barré 2005).

Depuis 2007, dans le cadre de notre recherche sur les abris sculptés et occupés du Magdalénien moyen, nous avons entrepris l'étude des graphismes pariétaux qui n'avaient jusqu'alors donné lieu à aucun travail analytique (Bourdier 2008). Suite à la reconnaissance de plusieurs ensembles graphiques successifs sur la frise, une collaboration a été engagée sur la révision du matériel (industries lithique et osseuse, art mobilier, parure) en complément de datations radiocarbones (Bourdier et al. soumis). Parallèlement, nous avons poursuivi l'étude du dispositif pariétal en nous intéressant aux blocs ornés de la collection Delage.

\section{1 - L'autre visage de l'art pariétal de Reverdit}

Au cours de ses fouilles et avant la découverte de la frise, F. Delage met au jour 19 blocs ornés, qu'il ne décrit que partiellement et dont il ne livre que trois clichés (Delage 1935). Pour la grande majorité, ils proviennent des déblais Reverdit et ne sont donc pas situés stratigraphiquement. Certains avaient même été utilisés dans l'appareillage d'un muret de pierres sèches que $A$. Reverdit avait édifié à l'extrémité de l'abri pour se débarrasser des matériaux encombrants (carnets de fouille inédits de F. Delage). Seul un support sculpté est issu « de la partie inférieure de la couche »(Delage 1935 : 306) sans préciser la couche incriminée (supérieure ou inférieure). Deux autres « gisaient, sans doute, [...] dans la partie supérieure du gisement »(Delage $1935:$ : 313).

Six blocs ornés sont toujours sur place tandis qu'un lot est conservé au Musée national de Préhistoire (8 dont 6 ornés). L'abri renferme des blocs provenant de plusieurs gisements du vallon (Reverdit, Castanet et Blanchard). 
Selon R. Castanet, seul le petit groupe rassemblé à l'extrémité gauche serait celui des gisements aurignaciens (4 dont 1 orné). Mais cette répartition n'est malheureusement pas assurée. En outre, le Musée national de Préhistoire possède des blocs ornés issus de plusieurs sites du vallon de Castel-Merle. Afin de prévenir tout risque de mélange, nous n'avons retenu que les éléments dont la traçabilité ne pouvait pas prêter à confusion : ceux publiés par $\mathrm{F}$. Delage et, au Musée national de Préhistoire, ceux portant le numéro d'inventaire des fouilles Delage $\left(E-1921^{2}\right)$. Même en considérant l'intégralité des blocs comme provenant bien de Reverdit, sept blocs manquent à l'inventaire de F. Delage. Nous avons donc finalement retenu sept blocs lesquels, à une exception peut-être, semblent correspondre à des fragments effondrés de la paroi, plutôt qu'à une ornementation au sol. En effet, l'incomplétude des figures et l'absence de cadrage sont considérés comme des éléments en faveur d'un dispositif effondré (Tymula 2002).

\section{2 - Les graphismes abstraits}

\section{Bloc n ${ }^{\circ} 1$ (MNP 1935-2-4)}

Le bloc (fig. 2) est un trapèze de $34,6 \mathrm{~cm}$ de longueur maximale et $28,3 \mathrm{~cm}$ de largeur maximale. Ses quatre bords fracturés sont abrupts. La face supérieure ${ }^{3} \mathrm{~s}^{\prime}$ inscrit sur deux plans : elle se coude à son extrémité gauche, ce changement d'orientation étant marqué par une arête. La surface est plane, attaquée sur tout le pourtour par un écaillage profond qui pourrait être corrélatif du détachement et de la chute du bloc. II entame presque toutes les cupules, ce qui irait dans le sens de graphismes pariétaux effondrés. Le reste de la face supérieure est marqué par une desquamation superficielle.

Le décor est constitué de sept cupules juxtaposées, de deux morphologies distinctes. Six présentent un fond arrondi. Quatre d'entre elles ont un gabarit similaire : entre 5 et $6 \mathrm{~cm}$ de diamètre et de 1 à $1,5 \mathrm{~cm}$ de profondeur. Leur contour est interrompu par l'écaillage périphérique du bloc, particulièrement les deux exemplaires de la partie inférieure. Les dimensions des deux autres sont respectivement plus réduites $(2,8 \mathrm{~cm}$ de diamètre) et plus grandes $(7 \mathrm{~cm})$. Au centre du support, la sixième cupule se singularise par son fond plat, tout à fait inhabituel, dans lequel s'impriment les stigmates rectilignes de coups métalliques récents. D'un diamètre nettement supérieur $(13 \mathrm{~cm})$, elle est aussi légèrement moins creusée. Les impacts du piquetage encore nettement visibles dans le fond des cupules, leur conférant un aspect grumeleux.

La distribution des cupules ne dessine pas de motif identifiable. Leur écartement varie. Seuls deux éléments sont jointifs, la répartition des autres n'indiquant pas de rythme précis. Pour autant, nous ne pensons pas que leur disposition soit aléatoire. Ainsi, un tracé en gravure large $(3 \mathrm{~cm})$ et profonde $(1,5 \mathrm{~cm})$ part de chacune des deux cupules jointives, de manière diamétralement opposée.
Ces traits témoignent d'une construction graphique manifeste dont la structure nous échappe cependant.

\section{Bloc nº 2 (MNP 1935-2-2)}

De forme trapézoïdale ( $40 \mathrm{~cm} \times 31 \mathrm{~cm}$ ), le bloc (fig. 3) se rétrécit fortement à l'une de ses extrémités. Ses bords sont abrupts. La face supérieure est fortement altérée par un écaillage couvrant une grande partie du support. Cette desquamation est particulièrement intense dans la moitié droite et dans la partie inférieure du bloc, surfaces érodées sur quelques centimètres. Ailleurs, le champ est plan mais tapissé d'écailles de petites dimensions relativement superficielles, lui donnant un aspect dentelé.

Trois cupules à fond arrondi creusent profondément le support $(2-2,5 \mathrm{~cm})$. Étroitement juxtaposées, elles sont alignées verticalement. La cupule centrale se distingue par un plus grand diamètre $(9 \mathrm{~cm})$. Les deux autres qui l'encadrent ont un même format $(6,5-7 \mathrm{~cm})$ créant ainsi un effet de symétrie. Des impacts de piquetage impriment toujours leur fond, en particulier celui de la cupule supérieure. Dans la moitié gauche, deux tracés piquetés, larges de 2,7 cm et profonds de $1,5 \mathrm{~cm}$, à section en $\mathrm{V}$ symétrique, complètent ce décor. Perpendiculaires, ils se rejoignent à leur extrémité. Ils sont accolés aux cupules sans apparaitre comme directement associés, ne recoupant pas leur contour. Dans l'alignement du tracé vertical, le bord supérieur du bloc marque une dépression qui pourrait être le vestige d'une troisième ligne.

\section{Bloc nº 3 (MNP 1935-2-1)}

Le bloc (fig. 4) est un rectangle de $44,6 \mathrm{~cm}$ de largeur maximale et $26,5 \mathrm{~cm}$ de hauteur maximale. Ses bords sont abrupts, excepté le bord gauche marqué par un rebord adouci. Comme sur le bloc $n^{\circ} 1$, un écaillage intense affecte tout le pourtour du support, notamment dans la partie droite très tourmentée. La surface interne plane n'est pas pour autant exempte de toute desquamation. Quelques écailles profondes l'entament principalement dans la moitié supérieure, celles de la partie basse étant en revanche superficielles. De larges plages cupulées, surtout concentrées au centre du support, pourraient indiquer un travail préparatoire d'aplanissement et de régularisation. Un dépôt superficiel grisâtre recouvre le tiers gauche du bloc, s'étendant surtout dans la zone inférieure.

Trois grandes cupules sont disposées sur le même axe horizontal, une première à l'extrémité gauche, les deux autres étroitement juxtaposées à l'extrémité droite. Bien que leur contour soit accidenté par la desquamation, leur gabarit est identique $(6 \mathrm{~cm}$ de diamètre, $1-1,5 \mathrm{~cm}$ de profondeur). Une quatrième, plus petite $(2 \mathrm{~cm})$, surplombe la seconde. Deux tracés gravés partent de la première cupule. L'un, vertical, est rapidement interrompu par l'écaillage du rebord. L'autre, horizontal, se scinde en deux lignes courbes qui rejoignent respectivement la deuxième

(2) Ce numéro d'inventaire est le même que celui de la série Delage de l'Institut de Paléontologie Humaine pour le compte duquel F. Delage avait réalisé ses fouilles (Delage 1935).

(3) Par commodité, nous appellerons « face supérieure » la face ornée et « face inférieure » celle qui lui est opposée. 


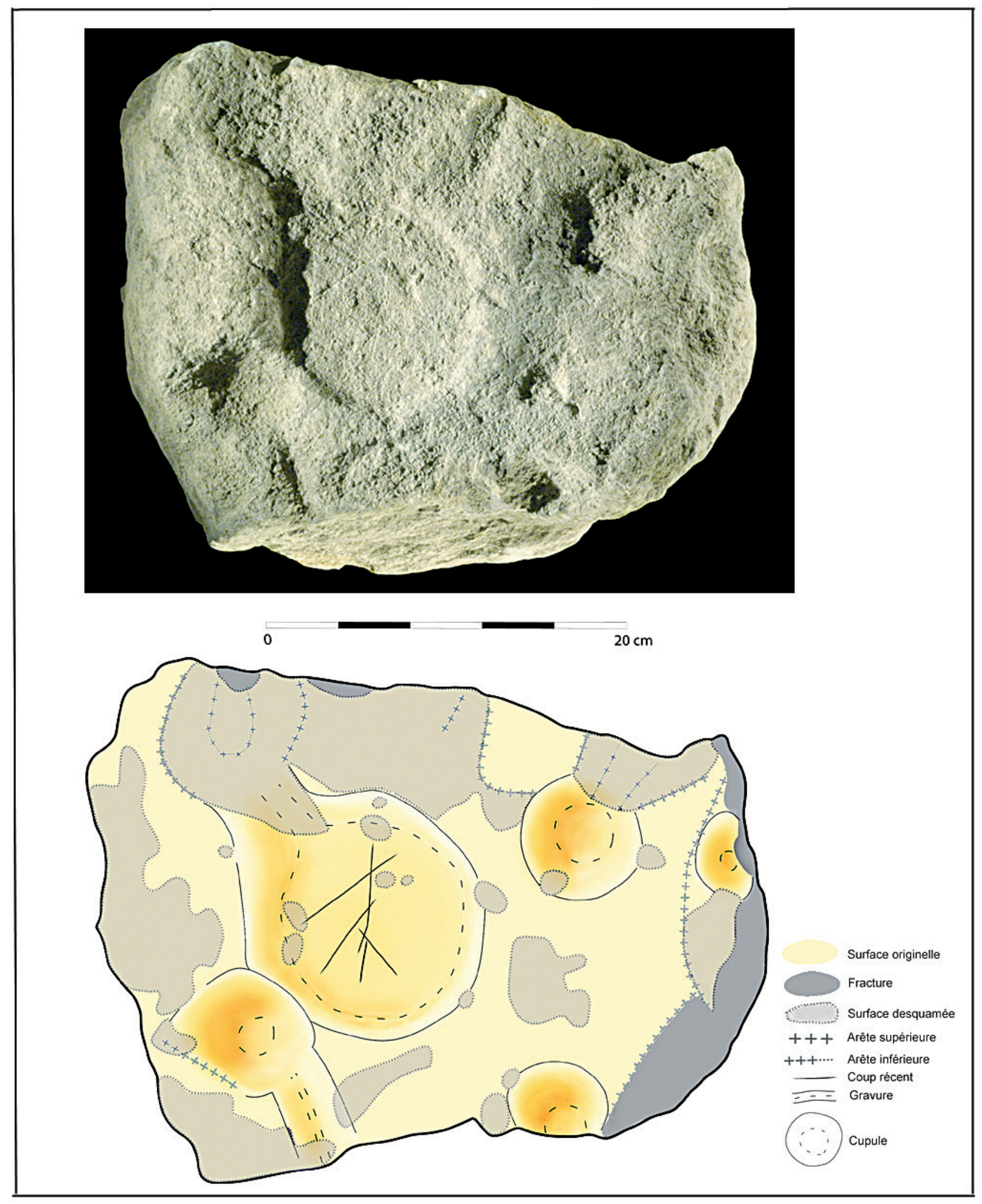

Figure 2 - Bloc orné $n^{\circ} 1$ (MNP 1935-2-4) : cliché et relevé synthétique. Cliché Ph. Jugie, MNP, relevé C. Bourdier.

Figure 2 - Decorated block $n^{\circ} 1$ (MNP 1935-2-4) : picture and synthetic tracing. Photo Ph. Jugie, MNP, tracing C. Bourdier. 


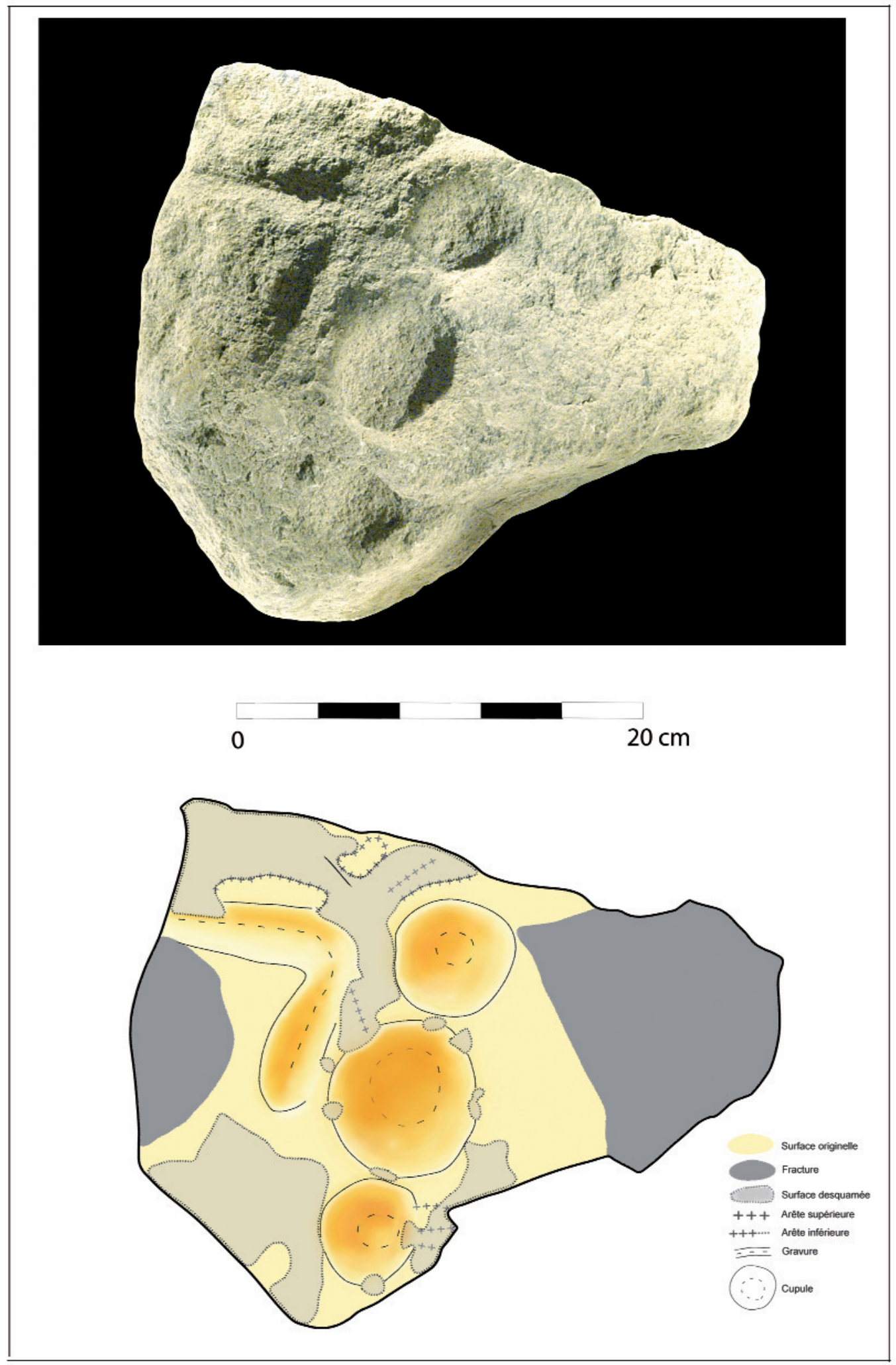

Figure 3 - Bloc orné $n^{\circ} 2$ (MNP 1935-2-2) : cliché et relevé synthétique. Cliché Ph. Jugie, MNP, relevé C. Bourdier.

Figure 3 - Decorated block $n^{\circ} 2$ (MNP 1935-2-2) : picture and synthetic tracing. Photo Ph. Jugie, MNP, tracing C. Bourdier. 


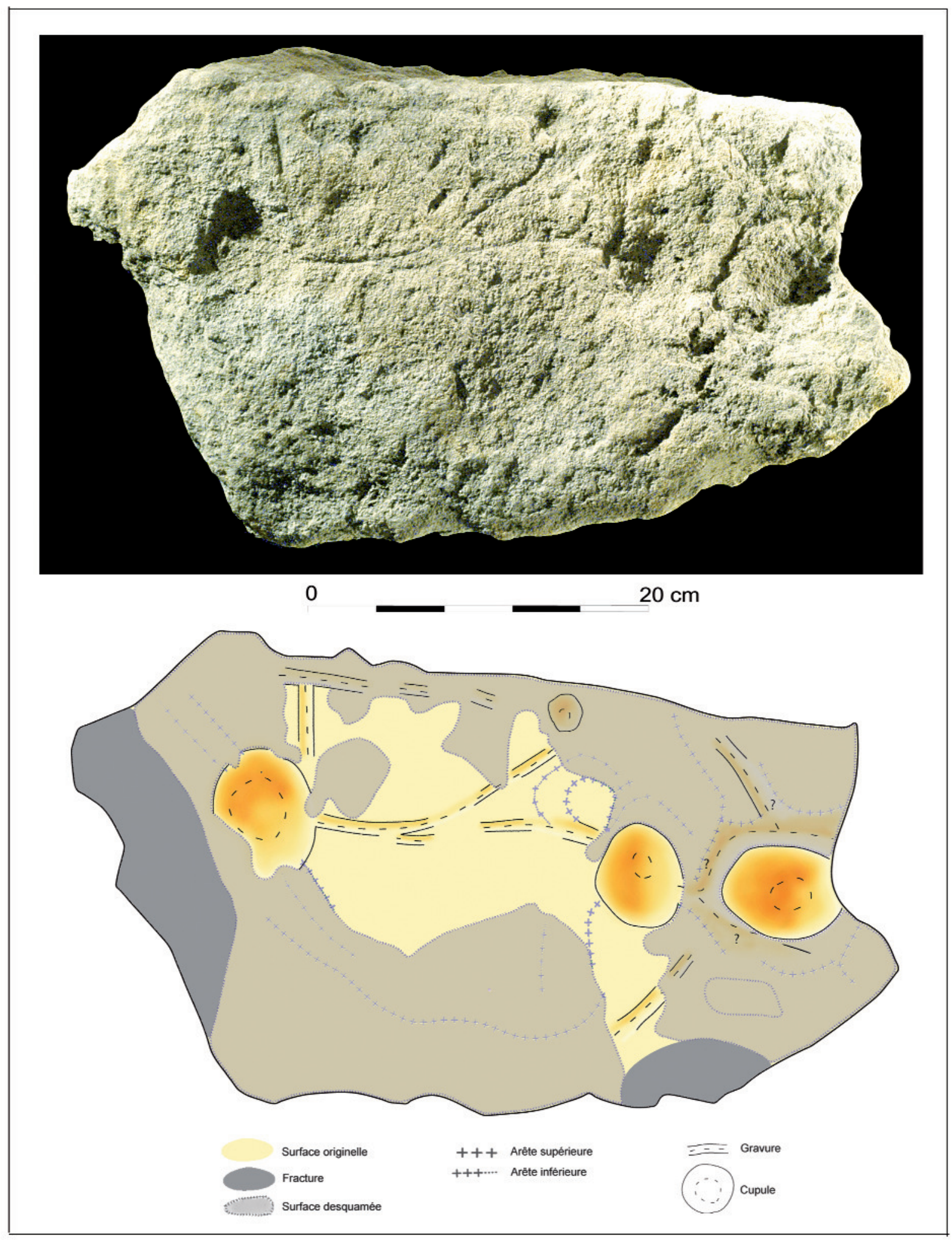

Figure 4 - Bloc orné $n^{\circ} 3$ (MNP 1935-2-1) : cliché et relevé synthétique. Cliché Ph. Jugie, MNP, relevé C. Bourdier.

Figure 4 - Decorated block $n^{\circ} 3$ (MNP 1935-2-1) : picture and synthetic tracing. Photo Ph. Jugie, MNP, tracing C. Bourdier. 


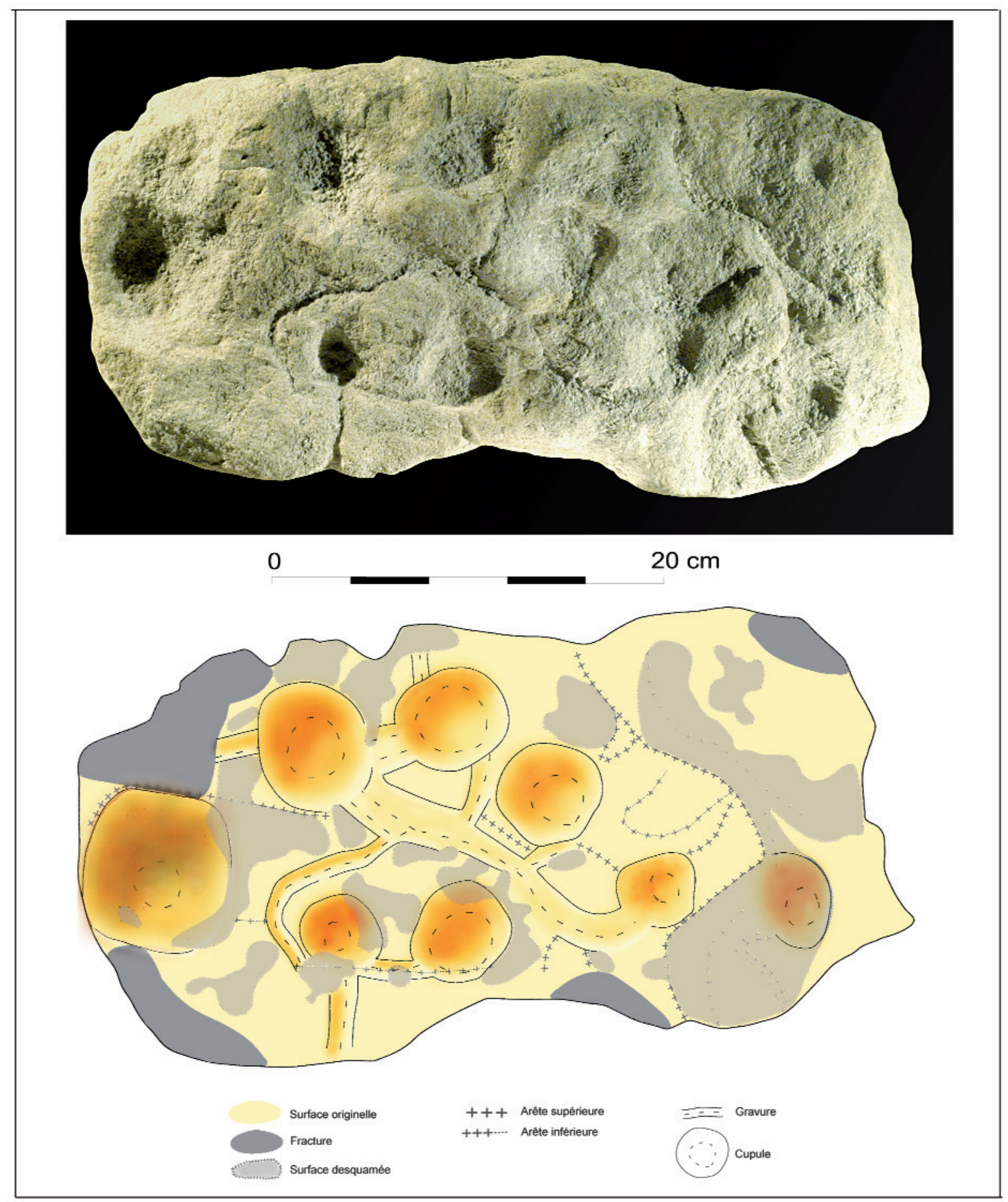

Figure 5 - Bloc orné $n^{\circ} 4$ (MNP 1935-2-5) : cliché et relevé synthétique. Cliché Ph. Jugie, MNP, relevé C. Bourdier. Figure 5 - Decorated block $n^{\circ} 4$ (MNP 1935-2-5) : picture and synthetic tracing @ MNP, photo Ph. Jugie, tracing C. Bourdier. 
et la quatrième cupule. L'altération du support empêche de voir si un tracé de ce type unit la deuxième cupule à la troisième. Un vestige subsiste au-dessus de la quatrième cupule, également fracturé par l'écaillage périphérique du bloc. D'autres tracés apparaissent plus hypothétiques : le long du bord supérieur ou depuis la première cupule vers le coin supérieur gauche. La gravure diffère de celle rencontrée sur les deux blocs précédents. Obtenue par un raclage simple, elle est ici moins accusée en largeur (3-4 mm) comme en profondeur (2-3 mm). Les cupules semblent ainsi organisées en réseau, reliées entre elles par des traits de gravure.

\section{Bloc n ${ }^{\circ} 4$ (MNP 1935-2-5)}

De forme rectangulaire $(46,6 \mathrm{~cm} \times 23,3 \mathrm{~cm})$, ce bloc (fig. 5 ) se distingue des autres supports par sa surface convexe et très accidentée. L'extrémité droite est nettement rabaissée. Elle correspond à un pan de fracture ancienne. Elle comporte une cupule mais pas de gravure associée. II est ainsi difficile de déterminer si cette fracture est antérieure ou postérieure au décor. Le reste du bloc est marqué par une forte courbure, avec un dénivelé de plus de $3 \mathrm{~cm}$ entre les moitiés supérieure et inférieure. II a subi une intense desquamation. Presque entièrement écaillée, sa surface a été profondément attaquée par endroits. Un encroûtement grisâtre s'est formé dans le fond de certaines dépressions.

Les sept cupules renforcent cet aspect très tourmenté. Trois formats coexistent : une particulièrement grande avec un diamètre de $9 \mathrm{~cm}$ creusant le support sur plus de $3 \mathrm{~cm}$, quatre d'un gabarit moyen $(5-6 \mathrm{~cm}$ de diamètre, $1,5-2 \mathrm{~cm}$ de profondeur) et deux légèrement plus petites $(3,5-4 \mathrm{~cm}$ de diamètre). Cinq sont disposées en arc de cercle dans la moitié supérieure du bloc, les deux dernières étant juxtaposées dans la partie basse. De nouveau, un entrelacs de traits gravés relie ces éléments. Réalisés en gravure large $(1-2 \mathrm{~cm})$ et appuyée $(0,5 \mathrm{~cm})$, leur perception varie fortement selon la puissance de la desquamation. S'ils sont bien marqués dans la moitié inférieure, ils apparaissent de manière uniquement vestigielle au centre du support. Des tracés courts et rectilignes cohabitent avec de longues lignes courbes selon la distance des cupules mises en relation.

\section{Une thématique pariétale élargie}

Ces blocs révèlent ainsi un décor géométrique complexe associant cupules et incisions. Les motifs diffèrent. La disposition des cupules varie : alignées sur un ou deux rangs, jointives ou plus largement juxtaposées, elles peuvent être raccordées ou non par des incisions. Leur morphologie est également changeante, en diamètre comme en profondeur. Le bloc $n^{\circ} 1$ combine ainsi une énorme cupule à fond plat très régulier et quatre cupules plus petites à fond arrondi. Excepté cet exemple à fond plat, l'origine naturelle ou anthropique de ces cupules n'est pas évidente au regard de leur état de conservation. Cependant, leur relative homogénéité et la régularité de leur espacement au sein de chaque bloc plaiderait en faveur d'une action anthropique, attendu que le support est globalement peu cupulé (paroi de l'abri). En outre, des stigmates de piquetage sont encore nettement visibles dans le fond de certaines. À défaut peut-être de ne pas les avoir toutes réalisées, les Paléolithiques ont utilisé des cupules naturelles qu'ils ont introduites comme motifs pariétaux en leur associant des traits de gravure.

Le dispositif pariétal de l'abri Reverdit comporte ainsi des thématiques figuratives et abstraites. L'absence de graphismes abstraits sur la paroi, si elle peut surprendre, ne nous semble pas pour autant révélatrice au regard de son intense desquamation. Sur les blocs, l'interruption fréquente des tracés au niveau des pans de fracture et la coexistence d'œuvres figuratives (voir ci-dessous) vont dans le sens de fragments pariétaux effondrés. À cause de ce morcellement, la comptabilisation des graphismes abstraits est illusoire: elle ne tiendrait d'aucune réalité, les motifs n'ayant pas pu être reconstitués et clairement individualisés. Leur fréquence n'est toutefois pas anecdotique.

Des raccords ont été testés entre les blocs du Musée national de Préhistoire et ceux conservés dans l'abri dont l'un notamment présente un motif similaire (fig. 6). Ils se sont malheureusement révélés infructueux. Une vision plus large de ces graphismes serait d'autant plus nécessaire que des décors cupulés sont connus dans les gisements aurignaciens et gravettiens du vallon de Castel-Merle. Néanmoins, leur structuration semble se détacher des " empreintes d'ours ", marqueurs culturels des sites ornés aurignaciens et gravettiens groupés autour de Sergeac et des Eyzies (Delluc et Delluc 1991) (fig. 7).

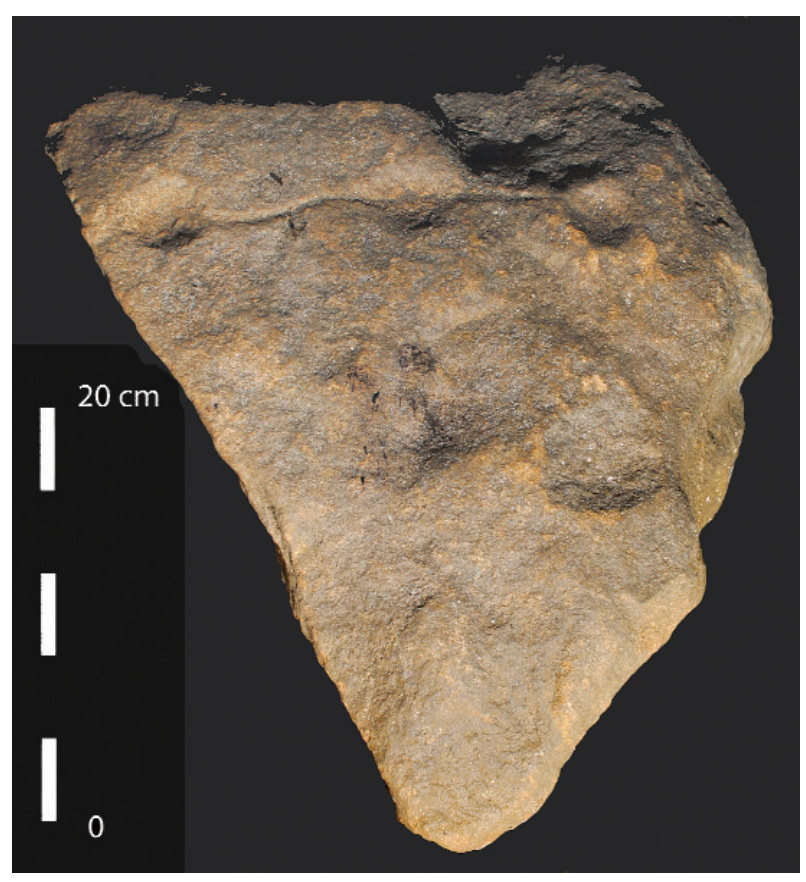

Figure 6 - Bloc orné de trois cupules reliées par un trait de gravure, conservé dans l'abri - origine inconnue (C) I. Castanet-Daumas, cliché C. Bourdier.

Figure 6 - Block ornate with three cupules linked by an engraved line, stored in the site - unknown origin (C) I. Castanet-Daumas, photo C. Bourdier. 
Bien qu'elle ne soit pas entièrement à rejeter en l'état des connaissances, l'hypothèse de récolte d'éléments ornés dans les gisements voisins par les Magdaléniens occupant l'abri Reverdit ne paraît pas être à privilégier. La perduration des motifs à cupules n'a au fond rien d'étonnant, la cupule étant l'un des motifs universels de l'art rupestre. En outre, ces motifs rappellent de petits signes quadrangulaires composés de cupules alignées et reliées par une fine incision, que l'on retrouve associés à des bas-reliefs au Roc-aux-Sorciers (Angles-sur-l'Anglin, Vienne), abri occupé et orné au Magdalénien moyen entre 15000 et 14000 BP (18 000 - 16000 cal. BP) (lakovleva, Pinçon 1997) (fig. 8).

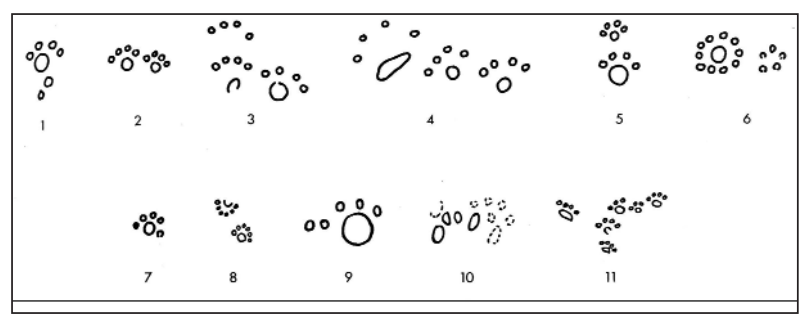

Figure 7 - Motifs en " empreinte d'ours » des gisements aurignaciens et gravettiens de la vallée de la Vézère ( Delluc et Delluc 1991.

Figure 7 - "bear spoor" patterns from the aurignacian and gravettian sites in the Vézère valley @ ( Delluc et Delluc 1991.

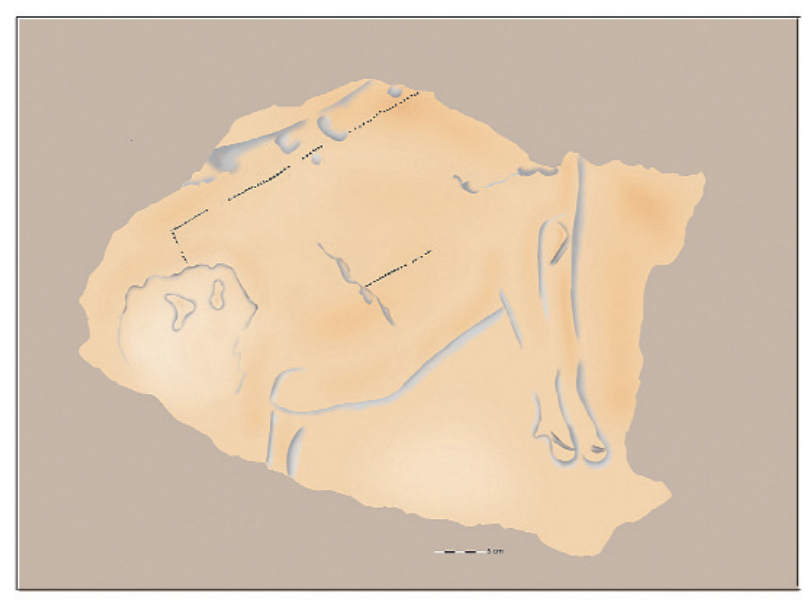

Figure 8 - Signe quadrangulaire composé de petites cupules alignées et reliées par une incision fine-Roc-aux-Sorciers (Angles-sur-l'Anglin, Vienne) () G. Pinçon, relevé O. Fuentes.

Figure 8 - Quadrangular sign, made of small cupules linked with a fine engraving - Roc-aux-Sorciers (Angles-sur-l'Anglin, Vienne) () G. Pinçon, tracing O. Fuentes.

\section{3 - Les sculptures animalières}

\section{Tête animale (MNP 1935-2-3)}

Le bloc (fig. 9) est un carré de $28,8 \mathrm{~cm}$ de largeur maximale, pour une hauteur maximale de $28,4 \mathrm{~cm}$. Ses fractures sont franches. La surface supérieure est érodée par une forte desquamation, particulièrement prononcée sur les rebords. Le support est attaqué par un écaillage intense, couvrant, auquel s'ajoutent quelques cupules profondes, de diamètre variable, concentrées dans la partie centrale. Bien que nous privilégions une origine naturelle de ces cupules, l'hypothèse de stigmates de martelage n'est pas à rejeter entièrement, ces éléments se situant sur les zones sculptées. Les volumes sont ainsi émoussés, compliquant la lecture du bloc. De manière générale, la distinction entre lignes naturelles et anthropiques est rendue délicate par cet état de surface très altéré.

Une tête animale $(16,5 \mathrm{~cm}$ de hauteur et $13,3 \mathrm{~cm}$ de largeur) en relief modelé $(0,5-1,5 \mathrm{~cm})$ est inscrite à l'intérieur de ce support. L'écaillage périphérique interrompt le départ du cou : s'agit-il effectivement d'un graphisme fracturé par la chute du bloc ? Ou d'une œuvre originellement fragmentaire, d'une tête isolée sur bloc, ce avec quoi le cadrage centré pourrait s'accorder? La figure a été mise en relief par creusement, plus appuyé pour le museau, puis le contour mis en forme par raclage dont le négatif est encore très net dans le fond de relief (chanfrein notamment). Le haut de relief n'est plus conservé. Le volume est actuellement plus marqué au niveau de la mandibule. Tout traitement interne (détails, modelé) est absent, probablement emporté par l'érosion. Seul un fond de trait courbe, remontant de la mandibule vers le sommet de la tête, signalerait le modelé de la joue.

La nature de l'animal ainsi n'est pas évidente à définir. Franck Delage y voyait un bison, identification à laquelle nous ne nous rangeons pas (Delage 1935). La morphologie générale ne concorde pas. Détachée de la masse du cou, la tête est longue et large. Elle est dotée d'un chanfrein rectiligne et d'un museau arrondi. Elle est dépourvue de barbe ou de barbiche. Un appendice court et pointu part du sommet du crâne : oreille, corne ? Juxtaposés à sa droite, deux tracés rectilignes en gravure profonde convergent en une pointe. Ils évoquent fortement une seconde oreille. La délinéation et les deux oreilles pointues dressées sur la tête rappellent les équidés, mais ces éléments restent trop ténus pour avancer une détermination. En avant du chanfrein, se discerne un autre trait en gravure profonde, courbe. Appartient-il à cette tête, ou est-il le vestige d'une seconde représentation?

\section{Arrière-train d'herbivore}

Conservé dans l'abri, le bloc (fig. 10) est un trapèze mesurant $45,8 \mathrm{~cm}$ de longueur maximale, sur une hauteur maximale de $48 \mathrm{~cm}$. Ses bords montrent des fractures abruptes. Ils sont sub-parallèles 2 à 2 , donnant au support une forme très régulière. Seul le rebord gauche est amputé dans son tiers supérieur par une fracture en arc de cercle. 


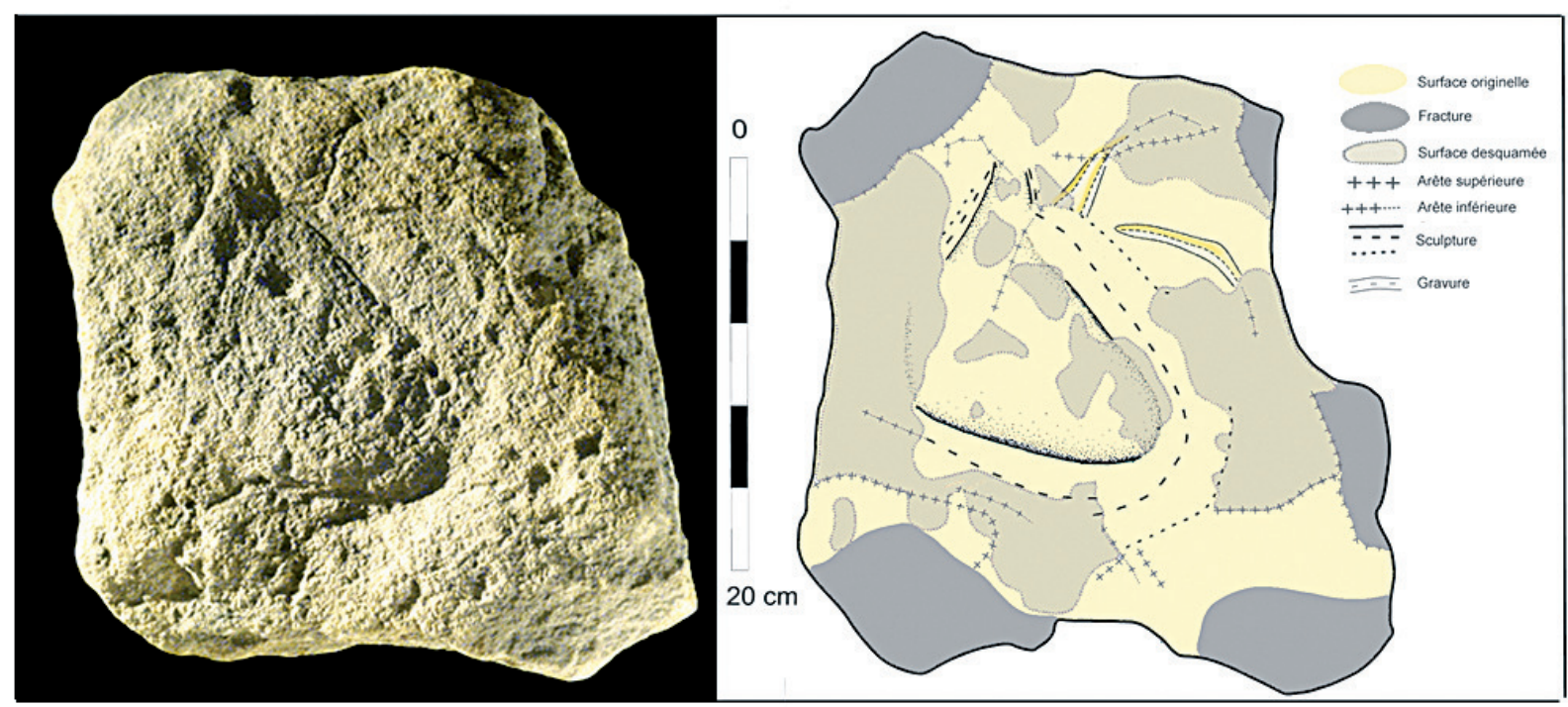

Figure 9 - Tête animale en relief modelé (MNP 1935-2-3) : cliché et relevé synthétique. Cliché Ph. Jugie, MNP, relevé C. Bourdier.

Figure 9 - Animal head in light relief (MNP 1935-2-3) : picture and synthetic tracing. Photo Ph. Jugie, MNP, tracing C. Bourdier.

La face inférieure est lardée de coups d'outils métalliques, traces d'un travail de régularisation lors de l'appareillage du bloc dans un mur suite aux fouilles d'A. Reverdit (carnets de fouille inédits de F. Delage). La face supérieure est assez bien préservée. Elle est relativement peu desquamée au regard des blocs précédents et des œuvres in situ. Quelques écailles minces se repèrent, principalement dans la moitié droite. Un encroûtement formé pendant la phase d'enfouissement recouvre la majeure partie du support. D'aspect grumeleux, présentant des vermiculations, il peut atteindre jusqu'à $3 \mathrm{~mm}$ d'épaisseur. Blanchâtre, il prend aussi une couleur marron au contact du sédiment.

Ce bloc est un fragment de bas-relief $(3-4 \mathrm{~cm})$ reproduisant un arrière-train. La ligne cervico-dorsale rectiligne est fracturée au niveau de la croupe. Les impacts du piquetage sont très visibles dans le fond de sculpture. La queue et la fesse manquent. Le contour de la cuisse épaisse est modelé, le postérieur étant brisé au niveau du jarret. De la ligne ventrale ne subsistent que quelques centimètres. Le volume du flanc et le galbe de la cuisse ont été soigneusement rendus. La technique de mise en relief ne peut malheureusement pas être précisée. L'épaisseur du flanc et le modelé robuste de la cuisse sont ceux d'un herbivore. Déterminé comme bovidé par F. Delage (1935), son morcellement nous interdit un diagnostic aussi précis : bovidé, cervidé, équidé ?

\section{Moitié inférieure de bison (MNP 35-1-1, MNP 35-1-2, MNP 35-1-3)}

Cette sculpture (fig. 11) est recomposée à partir de trois blocs. Deux morceaux comprenant les pattes, le ventre et le poitrail sont remontés par F. Delage (1935). À I'occasion d'un inventaire photographique au Musée national de Préhistoire, y est associé un troisième bloc portant la barbe et le départ du museau, ce remontage faisant d'ailleurs l'objet d'un court article présentant un premier croquis de lecture de la figure (Cleyet-Merle et al. 1994).
Les trois fragments possèdent des morphologies différentes. Ils ne recollent qu'au niveau de la sculpture et non sur la totalité de leurs pans de fracture. L'ensemble (84 $\mathrm{cm}$ de longueur sur $40 \mathrm{~cm}$ de hauteur maximale) montre donc un contour assez chaotique, très découpé. Le support est en grande partie fracturé : l'extrémité supérieure gauche et toute la partie basse sont des pans de fracture, soit près de la moitié de la surface. La surface originelle est ainsi réduite à une bande horizontale d'une vingtaine de centimètres de hauteur en moyenne, sur 80 $\mathrm{cm}$ de long. Elle est également entamée par quelques écailles profondes, principalement concentrées le long des fractures, ayant attaqué à divers endroits le haut de sculpture (extrémités des pattes, épaule, barbe). Néanmoins, le support bénéficie d'un bon état de conservation générale au sein de ce gisement.

Comme l'avait reconnu F. Delage, puis J.-J. Cleyet-Merle, $\mathrm{S}$. Madeleine et $\mathrm{Ph}$. Jugie, le contour inférieur d'un bison se détache, reconnaissable à la massivité du poitrail et à la barbe, ici reproduite par une excroissance angulaire pointée vers l'avant. La silhouette se suit parfaitement depuis les postérieurs jusqu'à la naissance du museau. Les membres modelés sont dessinés par paire : les postérieurs sont groupés, ramenés sous le corps, alors que les antérieurs sont décalés, l'antérieur droit sous lui et l'antérieur gauche campé. Leurs extrémités sont brisées. La ligne ventrale qui marque une forte pente vers les antérieurs est dotée d'un fourreau. Cette sculpture a bénéficié d'un traitement soigné et détaillé, avec notamment l'indication du volume de l'épaule et du pli du jarret. Ce bas-relief léger $(2-3 \mathrm{~cm})$ a été mis en relief par un dégagement plan très large, le support ayant été abaissé par piquetage dont les stigmates couvrent toute la zone exopérigraphique. La figure a ensuite été mise en forme par un piquetage plus précis, une bande cernant le contour de l'animal étant ainsi légèrement surcreusée sur 1 à $1,5 \mathrm{~cm}$. 


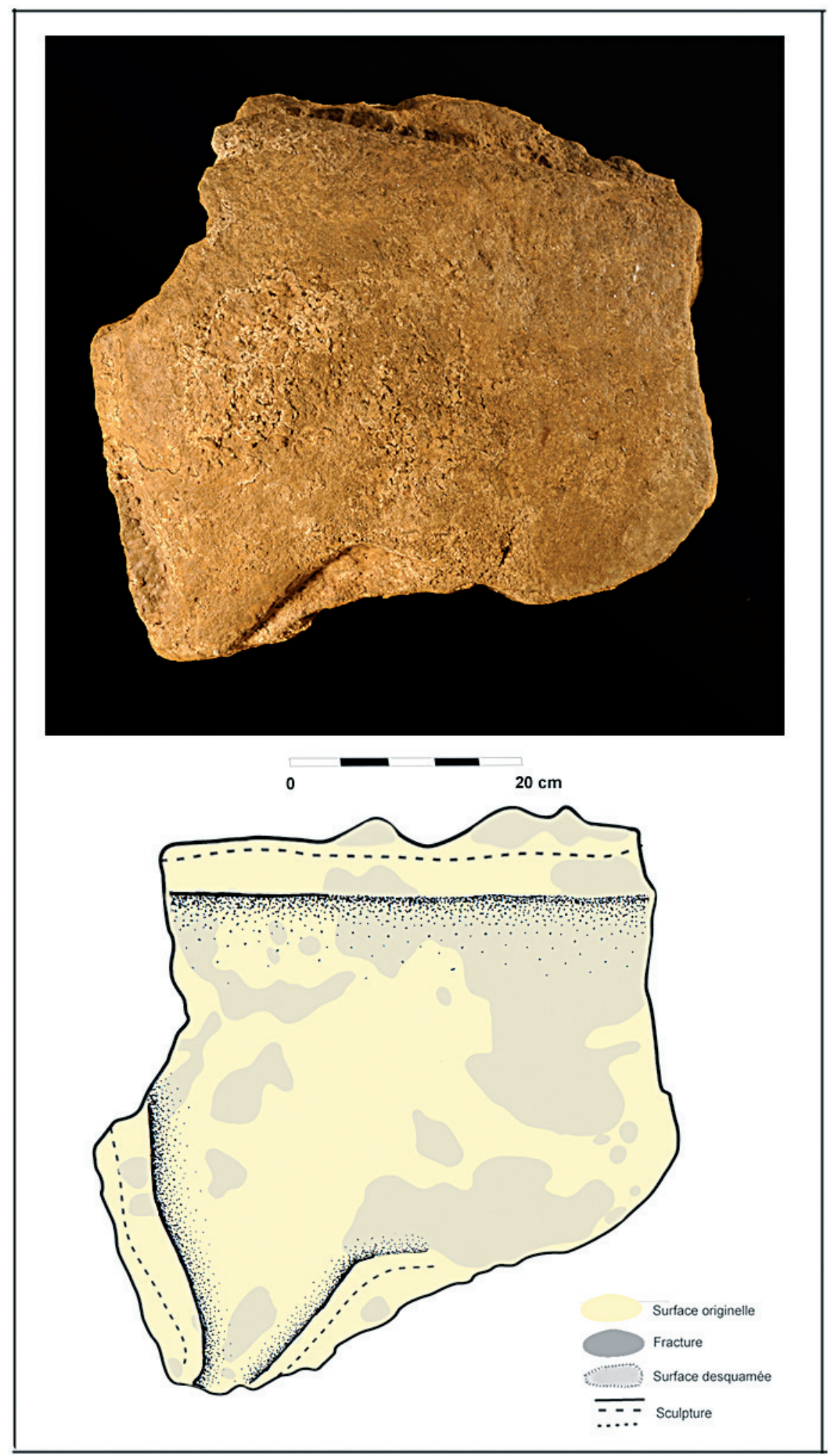

Figure 10 - Arrière-train fracturé d'herbivore en bas-relief : cliché et relevé synthétique (C) I. Castanet-Daumas, cliché A. Maulny, relevé C. Bourdier.

Figure 10 - Herbivore broken forelegs in low-relief: picture and synthetic tracing (C) I. Castanet-Daumas, photo A. Maulny, relevé C. Bourdier. 


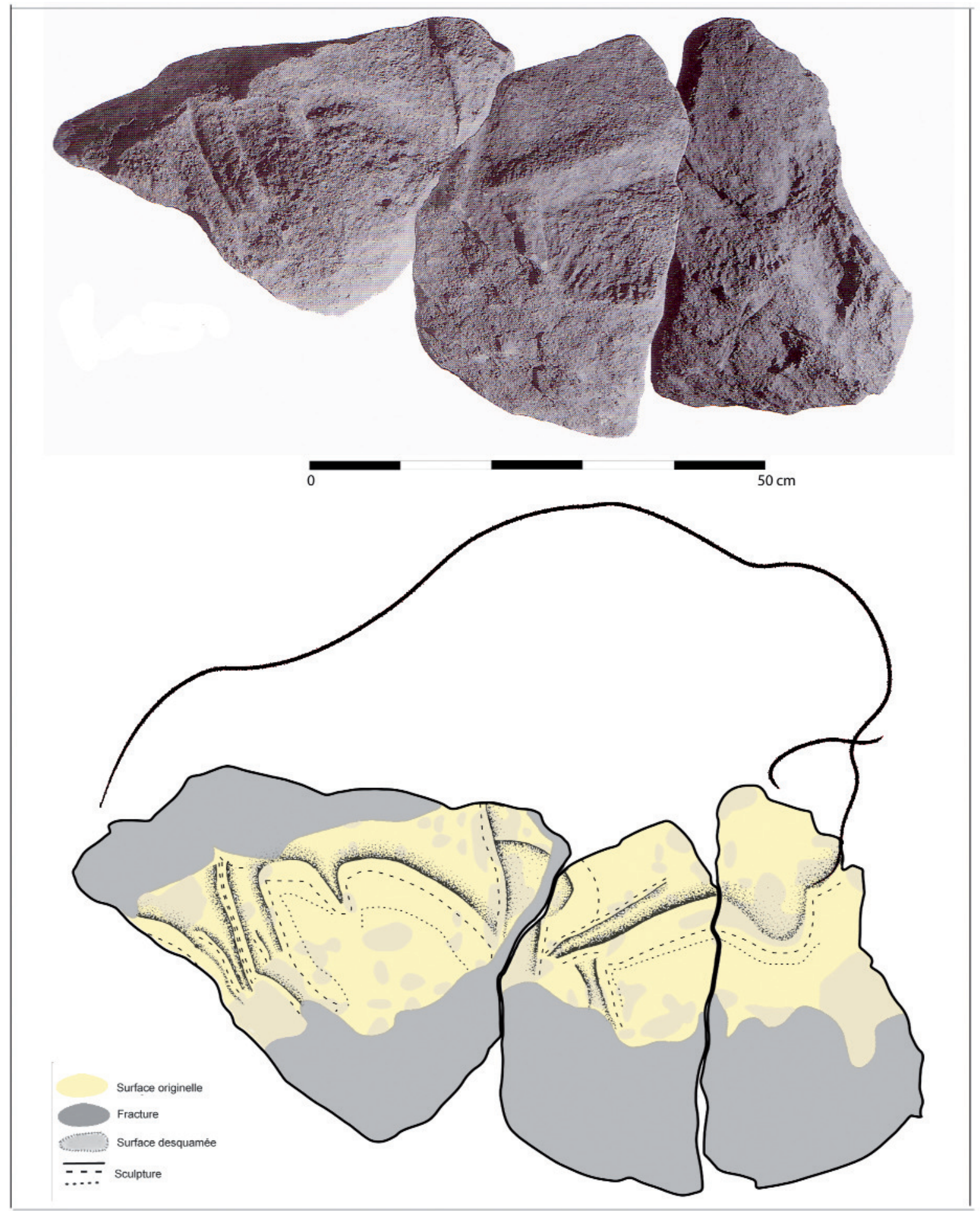

Figure 11 - Moitié inférieure de bison en bas-relief (MNP 35-1-1, MNP 35-1-2, MNP 35-1-3) : cliché et relevé synthétique (C) M.N.P., cliché B. et G. Delluc in Paillet 1999, relevé C. Bourdier.

Figure 11 - Bison lower part in low-relief (MNP 35-1-1, MNP 35-1-2, MNP 35-1-3) : picture and synthetic tracing (c) M.N.P., photo B. et G. Delluc in Paillet 1999, tracing C. Bourdier. 


\section{Une thématique animalière homogène}

La détermination des thèmes animaliers est ici compliquée par la fragmentation des sujets plus que par leur desquamation. En effet, les blocs n'ont pas évolué de la même manière que les graphismes in situ. Les processus érosifs qu'ils ont subis sont à la fois différents et moindres. Leur principale altération semble relever de leur fracturation, et de l'écaillage périphérique corrélatif, lors de leur détachement et de leur chute. Pris dans le remplissage, ils ont beaucoup moins souffert de la gélifraction, intensifiée dans l'abri par l'arrivée d'une source (Bourdier 2008). De même, leur surface ne présente pas de dégradation physico-chimique type mondmilch.

Ce morcellement des représentations contraint leur identification. Néanmoins, le bison, l'herbivore et l'équidé (?) sont des thèmes que l'on retrouve sur la frise, regroupant un herbivore (équidé ?), un cheval et trois bisons (Bourdier op. cit.). En outre, leur format - longueur de la tête animale, épaisseur du flanc de l'arrière-train d'herbivore, longueur du corps du bison - s'inscrit dans celui des œuvres in situ. Ces deux éléments soulignent l'homogénéité du dispositif pariétal, dans les thèmes et dans l'échelle de reproduction commune.

\section{4 - Une structuration pariétale complexe : la figure du bison}

Même si la fracturation des sujets sur paroi, comme sur blocs, réduit notre appréciation, le bison sur bloc se distingue des deux individus in situ, dans son traitement technique et formel (fig. 12). Les critères de comparaison sont pourtant limités. Le bas-relief léger mis en volume par un dégagement plan contraste avec les deux bas-reliefs épais $(10 \mathrm{~cm})$ de la frise, obtenus par un creusement en bassin très profond (Bourdier 2008). Les deux représentations in situ se caractérisent par une très forte hypertrophie antérieure, due à un rachis dorsal particulièrement développé en hauteur comme en largeur, à la morphologie carrée singulière, confondant garrot et chignon dans la même masse. Cet élément discriminant ne peut malheureusement pas être vérifié sur la figuration sur bloc. En revanche, poitrail et barbe diffèrent sensiblement. À la ligne descendant sur une barbe triangulaire verticale (frise) s'oppose le poitrail rectiligne, au fanon stylisé par une bande en relief, remontant sur la barbe ovalaire nettement détourée, pointée vers l'avant (bloc). En outre, bien que les mêmes volumes soient mis en valeur (épaule, bras), leur rendu diverge notablement, avec un relief très plat d'un côté (bloc) et un galbe très prononcé de l'autre (frise). Deux morphotypes de bison semblent ainsi coexister au sein du dispositif pariétal, le sujet sur bloc ne paraissant pas se conformer aux individus in situ. Contrairement à l'avis de F. Delage, la moitié inférieure sur bloc ne serait donc pas celle du second bison de la frise réduit au contour cervico-dorsal, même si leur gabarit concorde (Delage 1935). II n'est d'ailleurs pas évident que son contour inférieur n'eût jamais été réalisé, son tracé étant inachevé (Bourdier op. cit.).

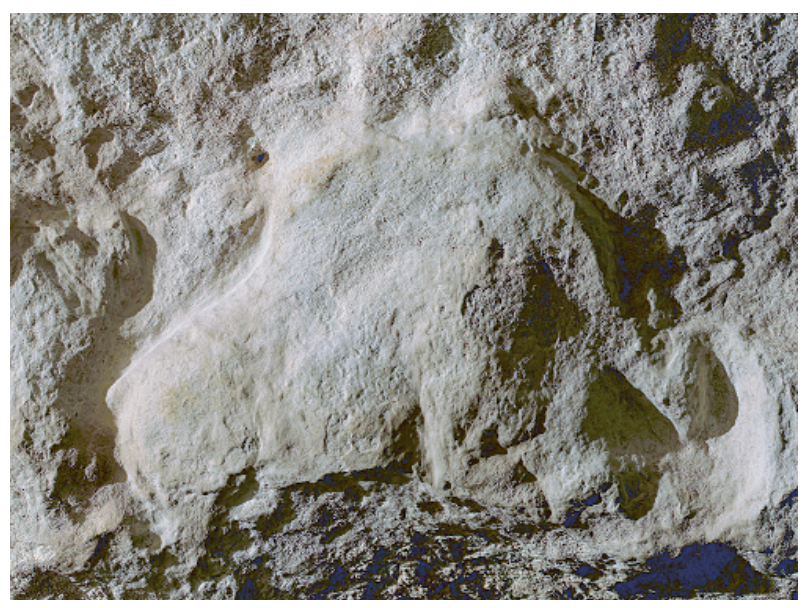

Figure 12 - Bison en bas-relief épais in situ (C) I. Castanet-Daumas, cliché A. Maulny.

Figure 12 - In situ bison in thick low-relief (C) I. Castanet-Daumas, photo A. Maulny.

L'analyse de la frise in situ a mis en avant deux ensembles graphiques successifs, illustrés par des retailles (Bourdier op. cit.). Un premier ensemble, dont ne subsistent que deux sculptures très fragmentaires de bisons (?), aurait cédé la place à un groupe de bas-reliefs associant deux bisons, un cheval et un herbivore (cheval ?). L'évolution du dispositif pariétal ne s'accompagnerait pas d'un changement thématique, peut-être tout au plus d'une diversification avec l'introduction du cheval. Si elle n'est pas motivée par la thématique, cette évolution pourrait s'expliquer par une transformation des codes graphiques, techniques et/ou formels. À ce titre, avec une technique et un morphotype distincts des deux individus in situ, le bison sur bloc nous paraît révélateur. Témoigne-t-il d'une phase de décor autre que celle des bisons de la frise ? Cette hypothèse a déjà été avancée par J.-J. Cleyet-Merle, S. Madeleine et Ph. Jugie qui y voyaient cependant un contraste plus fondamental entre un art pariétal (frise in situ) et un art semi-mobilier sur blocs (Cleyet-Merle et al. 1994).

Ce bison présente de nombreuses analogies avec les sculptures du Roc-aux-Sorciers. Sa comparaison avec l'un des bisons de la cave Taillebourg est particulièrement saisissante (fig. 13). Sa délinéation est tout à fait similaire : barbe ovalaire projetée vers l'avant, ligne ventrale descendante avec indication du fourreau pénien, pattes finement modelées avec mention du pli du jarret et figurées par paire dans une posture semblable. L'évocation stylisée du pelage du fanon sous la forme d'une bande en relief est, de plus, l'une des conventions typiques des bisons de ce site. Enfin, il s'inscrit dans des dimensions et dans des proportions très proches. Nous ne pouvons que regretter la fragmentation du sujet de Reverdit qui nous prive de précieux éléments quant au traitement de la tête et de la voussure dorsale, ou encore des sabots particulièrement détaillés au Roc-aux-Sorciers. 


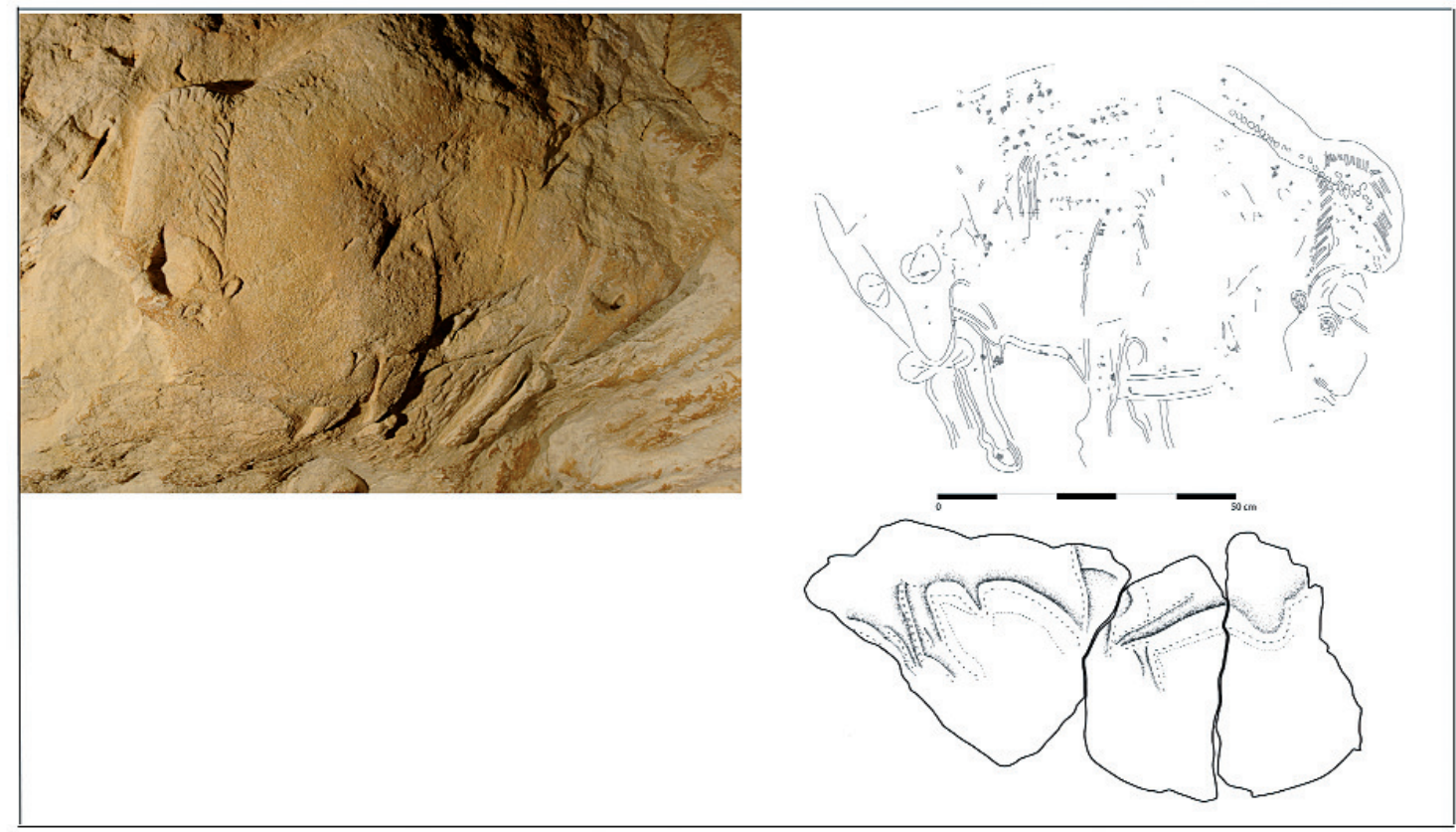

Figure 13 - Analogies des silhouettes du bison sur bloc et d'un bison du Roc-aux-Sorciers - 1/@ Ministère de la Culture et de la Communication, cliché G. Pinçon ; 2/@ G. Pinçon.

Figure 13 - Analogies between the outlines of the bison sculptured on block and a bison in Roc-aux-Sorciers rockshelter () 1/@ Ministère de la Culture et de la Communication, photo G. Pinçon ; 2/@ G. Pinçon.

\section{Conclusions}

Les blocs ornés viennent enrichir notre connaissance du dispositif pariétal de l'abri Reverdit tant au niveau de sa composition avec un nouveau registre thématique abstrait, que de sa structuration avec la coexistence de deux morphotypes de bison. II est néanmoins impossible d'intégrer ces graphismes au sein de la chronologie pariétale définie lors de travaux précédents, notamment les motifs abstraits qui n'ont aucun équivalent sur la paroi de l'abri. Le choix de la sculpture comme technique graphique, la similarité des thématiques animalières sur blocs et in situ ainsi que le recours à une échelle de reproduction commune soulignent en tous cas une certaine cohésion du dispositif pariétal. Les évolutions techno-formelles n'ont pas amené de bouleversements radicaux, mais ont plutôt l'air de s'être réalisées au sein d'un cadre général dont les grandes tendances graphiques ont été conservées. Enfin, une parenté apparaît avec le Roc-aux-Sorciers à travers la figure du bison et les motifs cupulés. Elle fournirait un élément d'attribution chronoculturelle au Magdalénien moyen, sans pour autant pouvoir préjuger de l'attribution de l'ensemble du dispositif pariétal.

\section{Remerciements}

Nous tenons à remercier chaleureusement Monsieur J.-J. Cleyet-Merle, conservateur en chef du Patrimoine, directeur du Musée national de Préhistoire et Madame I. Castanet-Daumas, propriétaire des gisements du vallon de Castel-Merle, de nous avoir autorisé l'accès aux blocs pour réaliser cette étude. Cette recherche a été menée dans le cadre de notre doctorat, réalisé grâce à l'allocation doctorale de la Région Poitou-Charentes.

\section{Références bibliographiques}

BARRÉ A. 2005 - Processus géologiques de formation du site de Reverdit (Sergeac, Dordogne), Mémoire de Master 2, Université Bordeaux 1, 51 p.

BOURDIER C. 2008 - La frise sculptée de l'abri Reverdit (Sergeac, Dordogne) : première approche analytique des œuvres. PALEO, 20, p. 23-46.

BOURDIER C., CHEHMANA L., PETILLON J.-M., VALLADAS $\mathrm{H}$. avec la collaboration de GAUTHIER C., KALTNECKER E. et MOREAU Ch., soumis - L'abri-sousroche de Reverdit (Sergeac, Dordogne) : l'apport d'une approche pluridisciplinaire à l'élaboration d'un nouveau cadre chrono-culturel. Actes du XXVII'̀me Congrés Préhistorique de France, Bordeaux-Les Eyzies, 30 mai-5 juin 2010.

CLEYET-MERLE J.-J., MADELEINE S. et JUGIE Ph. 1994 "À propos d'un bison gravé sur bloc de l'abri Reverdit à Sergeac (Dordogne) ». PALEO, 6, pp. 309-312.

DELAGE F. 1927 - Sergeac : un beau site périgourdin, un centre de recherches préhistoriques. Montignac : Imp. de la Vézère, $22 \mathrm{p}$.

DELAGE F. 1935 - Les Roches de Sergeac (Dordogne). L'Anthropologie, 45, p. 281-317.

DELLUC B. et DELLUC G. 1991 - L'art pariétal archaïque en Aquitaine. Paris : CNRS, XXVIIlème supplément à Gallia Préhistoire, $393 \mathrm{p}$. 
HARDY M. 1880 - « La station préhistorique des Roches, commune de Sergeac ». B.S.H.A.P., 7, p. 110-113.

IAKOVLEVA L., PINÇON G. 1997 - Angles-sur-l'Anglin (Vienne), La Frise sculptée du Roc-aux-Sorciers. Paris : Coédition Comité des Travaux Historiques et Scientifiques et Réunion des Musées Nationaux, 168 p. ill.

PAILLET P. 1999 - Le bison dans les arts magdaléniens du Périgord. Paris : CNRS Ed., 475 p. ill.

REVERDIT A. 1878 - «Stations et traces des temps préhistoriques dans le canton de Montignac-sur-Vézère ». B.S.H.A.P. 5, p. 384-419.

REVERDIT A., 1882 - Station des Roches, commune de Sergeac. Toulouse : Durand, Fillous et Lagarde, $45 \mathrm{p}$.
ROBIN D. 1985 - Abri Reverdit. Commune de Sergeac (Dordogne). Rapport de sondage 1985. S.R.A. Aquitaine, $10 \mathrm{p}$.

ROBIN D. 1986a - Sergeac, Castel-Merle, abri Reverdit. Gallia Préhistoire, 29, p. 240-241.

ROBIN D. 1986b - Abri Reverdit. Commune de Sergeac (Dordogne). Rapport de fouille de sauvetage 1986. S.R.A. Aquitaine.

ROBIN D. 1987 - Abri Reverdit. Commune de Sergeac (Dordogne). Rapport de fouille 1987. S.R.A. Aquitaine.

TYMULA S. 2002 - L'art solutréen du Roc de Sers (Charente). Paris : document d'archéologie française, $285 \mathrm{p}$. 